\title{
A Genomic Perspective of Protein Kinases in Plasmodium falciparum
}

\author{
Anamika, N. Srinivasan, ${ }^{*}$ and A. Krupa \\ Molecular Biophysics Unit, Indian Institute of Science, Bangalore, India
}

\begin{abstract}
Protein kinases are central to regulation of cellular signaling in the eukaryotes. Wellconserved and lineage-specific protein kinases have previously been identified from various completely sequenced genomes of eukaryotes. The current work describes a genome-wide analysis for protein kinases encoded in the Plasmodium falciparum genome. Using a few different profile matching methods, we have identified 99 protein kinases or related proteins in the parasite genome. We have classified these kinases into subfamilies and analyzed them in the context of noncatalytic domains that occur in these catalytic kinase domain-containing proteins. Compared to most eukaryotic protein kinases, these sequences vary significantly in terms of their lengths, inserts in catalytic domains, and co-occurring domains. Catalytic and noncatalytic domains contain long stretches of repeats of positively charged and other polar amino acids. Various components of the cell cycle, including 4 cyclin-dependent kinase (CDK) homologues, 2 cyclins, 1 CDK regulatory subunit, and 1 kinase-associated phosphatase, are identified. Identification of putative mitogen-activated protein (MAP) Kinase and MAP Kinase Kinase of $P$. falciparum suggests a new paradigm in the highly conserved signaling pathway of eukaryotes. The calcium-dependent kinase family, well represented in $P$. falciparum, shows varying domain combinations with EF-hands and pleckstrin homology domains. The analysis reveals a new subfamily of protein kinases having limited sequence similarity with previously known subfamilies. A new transmembrane kinase with 6 membranespanning regions is identified. Putative apicoplast targeting sequences have been detected in some of these protein kinases, suggesting their export to the apicoplast. Proteins 2005;58:180-189.

๑ 2004 Wiley-Liss, Inc.
\end{abstract}

Key words: genome analysis; Plasmodium falciparum; protein domains; protein kinases; signal transduction

\section{INTRODUCTION}

The processes of cellular proliferation and differentiation are tightly regulated in the eukaryotes by various signaling proteins. The activities of these proteins are in turn controlled by reversible phosphorylation catalyzed by protein kinases $(\mathrm{PKs})$ and phosphatases. A catalytic core of approximately 300 amino acid residues of $\mathrm{PKs}$ is involved in the phosphorylation of substrate proteins at Ser/Thr/ Tyr residues in eukaryotes. ${ }^{1}$ The conserved 3-dimensional (3D) structure of the kinase catalytic domain is bilobed, with substrate and adenosine triphosphate (ATP) binding sites located at the cleft between the two lobes. ${ }^{2}$

Specific features enabling the recognition of substrates, translocation of the enzymes to their substrate sites, and distinct modes of regulation lead to the functional diversity of PKs. ${ }^{3}$ The modular organization of PKs is hence an essential feature guiding them to their appropriate targets. The repertoire of PK in eukaryotes greatly enhances the understanding of the influence of phosphorylation in the growth and development of the organism.

The life cycle of Plasmodium falciparum is complex, involving a vertebrate host (man) and a mosquito vector. The intricate modalities of adaptation and survival strategies in dual hosts therefore involve communication between the proteins of the parasite and the host proteins. $\mathrm{PKs}$ that act as switches in various eukaryotic signaling networks are hence implicated to influence their diverse morphological stages in both the hosts.

The role of phosphorylation in various stages of parasite development and infection of the host is well documented. ${ }^{4-6}$ The invasion and intraerythrocytic development of $P$. falciparum has been shown to be inhibited by PK inhibitors. ${ }^{4}$ Phosphorylation of proteins subsequent to infection of erythrocytes is suggested to be influenced by $P$. falciparum and host erythrocyte skeletal protein interactions. ${ }^{5}$ Host erythrocytic membrane proteins are known to be phosphorylated by calcium-dependent protein kinases (CDPKs) of P. falciparum. ${ }^{6}$ Phosphorylation of endogenous substrates and the host proteins by the PKs of parasites has been previously observed in the asexual life cycle of $P$. falciparum. ${ }^{7}$

The Supplementary Materials referred to in this article can be found at http://www.interscience.wiley.com/jpages/0887-3585/suppmat/index.html

Grant sponsor: Fellowship from the Council of Scientific and Industrial Research, India (to A. Krupa). Grant sponsor: International Senior Fellowship in Biomedical Sciences (to N. Srinivasan) by the Wellcome Trust, UK, Computational Genomics Project, supported by the Department of Biotechnology, India, and the NMITLI project supported by CSIR, India.

*Correspondence to: N. Srinivasan, Molecular Biophysics Unit, Indian Institute of Science, Bangalore 560 012, India. E-mail: ns@mbu.iisc.ernet.in

Received 8 March 2004; Accepted 26 June 2004

Published online 28 October 2004 in Wiley InterScience (www.interscience.wiley.com). DOI: 10.1002/prot.20278 
Here we present a detailed analysis on the repertoire of the eukaryotic PKs in the completely sequenced genome of $P$. falciparum. An attempt has been made to identify all PK-like sequences encoded in the parasite genome using various sequence and profile search methods. The Hanks et al. ${ }^{1}$ classification of $\mathrm{PKs}$ and analysis of their domain organization has been further carried out in order to gain insight into their biological roles. We hope that the PK complement of the parasites, as identified in the current analysis, therefore leads to further studies aimed at understanding the mechanism of regulation of various cellular processes and the host-parasite interactions.

\section{MATERIALS AND METHODS}

The complete set of predicted protein sequences from the open reading frames (ORFs) of the $P$. falciparum genome ${ }^{8}$ has been obtained from Plasmodb (release 3.3) [http:// plasmodb.org/]. ${ }^{9}$ The genes encoded by $P$. falciparum and reported in this release have been predicted using various gene prediction methods, ${ }^{10,11}$ such as GlimmerM,${ }^{12}$ FullPhat, ${ }^{13}$ and Genefinder (C. Wilson, L. Hilyer, and P. Green, unpublished results). The accuracy of gene predictions made by multiple methods optimized for $P$. falciparum is therefore considered quite significant. ${ }^{11}$ We have surveyed the genome for Ser/Thr and Tyr kinases using sensitive sequence profile matching algorithms.

The sequence search tools and other strategies for domain identification and subfamily classification used in this study are same as those used in our earlier analysis of other genomes. ${ }^{14,15}$ Briefly, we have employed multiple, sensitive sequence search and analysis methods such as PSI-BLAST, ${ }^{16}$ RPS-BLAST, ${ }^{17}$ and HMMer, ${ }^{18}$ which matches hidden Markov models (HMMs) to identify PK catalytic domains and their co-occuring domains. An assessment of the reliability of domains assigned to kinasecontaining gene products, at various $E$-values using the HMMer method, has been made by consulting previous studies $^{19}$ and those carried out in our group by considering a data set of proteins of known 3D structures ${ }^{20,21}$ (N. Mhatre, B. Anand, and N. Srinivasan; unpublished results). According to these studies, searches performed using HMMer with an $E$-value cutoff of 0.01 or lower have been shown to nearly eliminate any spurious hits (near $100 \%$ specificity), without significant loss in the number of true homologues ( $80 \%$ sensitivity). In addition, search procedures such as PSI-BLAST have been used to detect sequences homologous to the kinase catalytic domain using an $E$-value cutoff of 0.0001 that has been previously benchmarked. ${ }^{22}$ Hits lacking significant sequence similarity with the query have been further examined manually and assessed by various fold prediction methods for the compatibility of the query sequence with the $3 \mathrm{D}$ fold of Ser/Thr and Tyr kinases.

The final data set of 99 PK-like sequences has been obtained from the compilation of hits obtained using various procedures. An all-against-all comparison of kinase domains in 99 sequences, using BLAST, was made in order to eliminate redundant sequences, which are indicated by $90 \%$ or greater sequence identity. The highest sequence identity shared between any 2 sequences in the data set does not exceed $50 \%$, suggesting that the data set is devoid of redundant sequences.

The expression profiles of various $P$. falciparum gene products that have been derived using various large-scale microarray data and other proteomic analyses ${ }^{23-25}$ have been consulted to confirm the expression of PK-encoding ORFs identified in the current analysis. The PK gene products that were not listed in any of the large-scale expression studies have been surveyed in the database of Expressed Sequence Tags of $P$. falciparum using tblastn, ${ }^{16}$ with an $E$-value cutoff of 0.01 . Microarray and massspectrometric expression data reveal 81 of the PKencoding genes as potentially expressed ORFs in $P$. falciparum.

\section{Classification of $P$. falciparum Protein Kinases Into Subfamilies}

Reverse PSI-BLAST (RPS-BLAST) was used to search each of the 99 PK-like sequences identified in the $P$. falciparum genome as a query against the database containing 55 position-specific matrices (PSSMs) created for the various subgroups of PKs in each of the subfamilies. The query kinase sequence was associated with its nearest subfamily based on the extent of sequence similarity, as described in our previous analysis. ${ }^{14}$

CLUSTALW has been used to align the catalytic domains of $61 \mathrm{PKs}$ of $P$. falciparum that were associated with known subfamilies. ${ }^{26}$ MEGA version $2.1^{27}$ was further used to generate the dendrogram showing the various subfamilies of PKs.

Domain assignment to the noncatalytic regions of the kinase-containing genes has been carried out using HMM search methods by querying each of the kinase domaincontaining sequences against the 6190 protein family HMMs available in the Pfam database. ${ }^{28}$ Transmembrane segments were detected using TMHMM. ${ }^{29}$ The PATS program $^{30,31}$ has been used to identify the putative signal peptide and transit peptide located at the N-terminal ends of the Plasmodium PKs.

\section{Phylogenetic Analysis of the New Subfamily of Protein Kinases in $P$. falciparum}

In order to identify the closely related homologues of the new subfamily of PKs of $P$. falciparum in other organisms, PSI-BLAST searches on the nonredundant database [NRDB available at the National Center for Biotechnology Information (NCBI)] of proteins have been carried out using the kinase catalytic domain of each member of the new kinase subfamily as a query. The protein sequences matching the query with highest sequence identity and with a reliable $E$-value $(<0.0001)$ have been further extracted from each of these searches to collect the close homologues of the new kinases. Multiple sequence alignment of the kinase catalytic domains of the new members and their most closely related homologues has been further carried out using CLUSTALW. ${ }^{26}$ The multiple sequence alignment has been further used to generate a sequence similarity-based dendrogram using MEGA software. $^{27}$ 


\section{RESULTS AND DISCUSSION Distribution of Protein Kinases in the P. falciparum Genome}

The genome of the malaria parasite encodes 99 PK-like sequences and 3 lipid kinases. A list of all these sequences with classification and domain combinations is available (http://hodgkin.mbu.iisc.ernet.in/king/cgi/eukaryotes_list. cgi?plasmodium falciparum) as a part of KinG database of PKs encoded in genomes. ${ }^{15}$ Among the 99 gene products containing PK domains encoded by the $P$. falciparum genome, 82 gene products correspond to the genes expressed in $P$. falciparum, as suggested by earlier studies $^{23-25}$ (81 PK gene products) and by their presence in the database of $P$. falciparum expressed sequence tags (ESTs; 1 PK gene product). The search in a database of $P$. falciparum ESTs also identified those corresponding to 5 of the remaining gene products with significant $E$-values ( $<$ $10^{-36}$ ) over identical regions of less than 150 amino acids. The remaining 12 PK domain-containing gene products currently lack information regarding their expression in the parasite; hence, their expression profile is not clear. However, the translated ORFs of $P$. falciparum that have been surveyed in the current analysis has been predicted using multiple gene prediction software. A further characterization of their cellular expression requires experimental validation. These results therefore suggest the expression of an overwhelming majority of PK gene products encoded by the parasite (Supplementary Information 1).

Among the 99 PK-like sequences, the catalytic aspartate is conserved in 90 sequences, while the ATP-anchoring glycine-rich loop contains at least 1 glycine in 65 kinase domain-containing gene products. The gene products lacking the catalytic base (Asp) are unlikely to possess phosphotransferase activity. The roles of such PK-like sequences in $P$. falciparum are currently unclear. However, such kinase homologous domains (KHD) lacking functionally critical residues have been implicated in the regulation of various signaling pathways. ${ }^{32-34}$ The catalytic base residue is lacking also in 2 of the phospholipid kinase-like sequences (PFE0485w, PFD0965w). The only phospholipid kinase (PFE0765w) with well-conserved functional residues has a phosphoinsositide kinase accessory (PIKa) domain located to the N-terminal of the catalytic domain. The $90 \mathrm{PKs}$ of $P$. falciparum with conserved catalytic aspartate, and hence expected to be functional, are referred to as putative kinase-like sequences (PKLSs) in the subsequent sections. Furthermore, the expression of 82 gene products among the identified PKLSs has been reported in large-scale proteomic and microarray analyses. $^{23-25}$ The Lys-Glu salt bridge ${ }^{3}$ localizing the gamma phosphate is not well conserved in 13 PKLSs. The variations thus observed in the key nucleotide-binding residues suggest a high divergence of the Plasmodium kinases compared to the other eukaryotic kinases known so far.

The phosphorylation of the activation segment is required for activation in most PKs that contain an arginine preceding the catalytic aspartate. These kinases are also referred to as "RD" kinases. ${ }^{4}$ The $P$. falciparum genome encodes a total of 55 "RD" kinases, suggesting a potential requirement for phosphorylation in their activation loop for regulation.

In comparison with the other eukaryotes, ${ }^{14,35-39}$ the $P$. falciparum genome represents a highest fraction of PKs with large extensions either at the $\mathrm{N}$-terminal or $\mathrm{C}$ terminal to the kinase catalytic domain, giving rise to gene products with greater than 1500 amino acids. The largest of these PKs is 4044 amino acids long (MAL13P1.278), with its kinase domain bearing highest sequence similarity to the protein kinase A (PKA)-like and the CDPKs. The $\mathrm{N}$ - and the $\mathrm{C}$-terminal regions of this gene product share, respectively, $33 \%$ and $45 \%$ sequence identity over a stretch of about 450 and 100 amino acid residues with a protein from Plasmodium yoelli yoelli (gi|23489871), which is annotated as a chloroquine resistance marker protein. However, these regions lack similarity with any other protein domain of known function.

\section{Classification of the $\boldsymbol{P}$. falciparum Protein Kinases}

Based on the amino acid sequence of the catalytic domain, $P$. falciparum kinases could be classified into various subfamilies as described by Hanks et al. ${ }^{1}$ (Fig. 1). More than half the number of PKLS (61) could be placed into the known subfamilies. ${ }^{1}$ The distribution and the diversity of the various subfamilies are discussed below.

\section{Calcium-Dependent Kinases (CDPKs)}

The CDPKs are a largely represented subfamily of PKs in the parasite, consisting of 21 PKLSs with varying domain combinations (Fig. 2). Some of these kinases have been previously studied. ${ }^{6}$ The well-characterized calciumbinding modules, EF-hands, are tethered to the catalytic domains of a subset (7) of these kinases. The kinase domain of these PKLSs is highly similar to the CAMK-1 subgroup of kinases (about $32 \%$ of sequence identity) that includes the plant CDPKs and $\mathrm{Ca}^{2+} /$ calmodulin-dependent kinases found in other eukaryotes. The number of the EF-hands in CDPKs of the parasite varies from 2 to 4. Plasmodium is the only other genus apart from plants, known so far, that encodes the EF-hand-containing PKs, and their activity is shown to be sensitive to $\mathrm{Ca}^{2+}$ concentration. ${ }^{40,41}$ These kinases are known to be regulated by the autoinhibitory region located between the $\mathrm{N}$-terminal catalytic domain and the C-terminal EF-hand modules. The $P$. falciparum also encodes two PKLSs that differ from the family of CDPKs described above by having $2 \mathrm{EF}$ hands each, to the $\mathrm{N}$-terminal and C-terminal of the kinase domain (PF11_0239), and another variant with 2 EF-hands at the N-terminal of the kinase domain (PF11_0242). The latter also has an unusual domain combination (Fig. 2), with the 3 GETHR repeats and the pleckstrin homology $(\mathrm{PH})$ domains occurring between the N-terminal EF-hands and the kinase catalytic domain, and has also been identified recently by another group. ${ }^{42}$ This CDPK is likely to localize at the erythrocytic membrane to utilize the sequestered $\mathrm{Ca}^{2+}$ reserves, resulting in its activation and subsequent phosphorylation of the red blood cell (RBC) membrane proteins that alters their permeability and fragility. The occurrence of the $\mathrm{PH}$ 


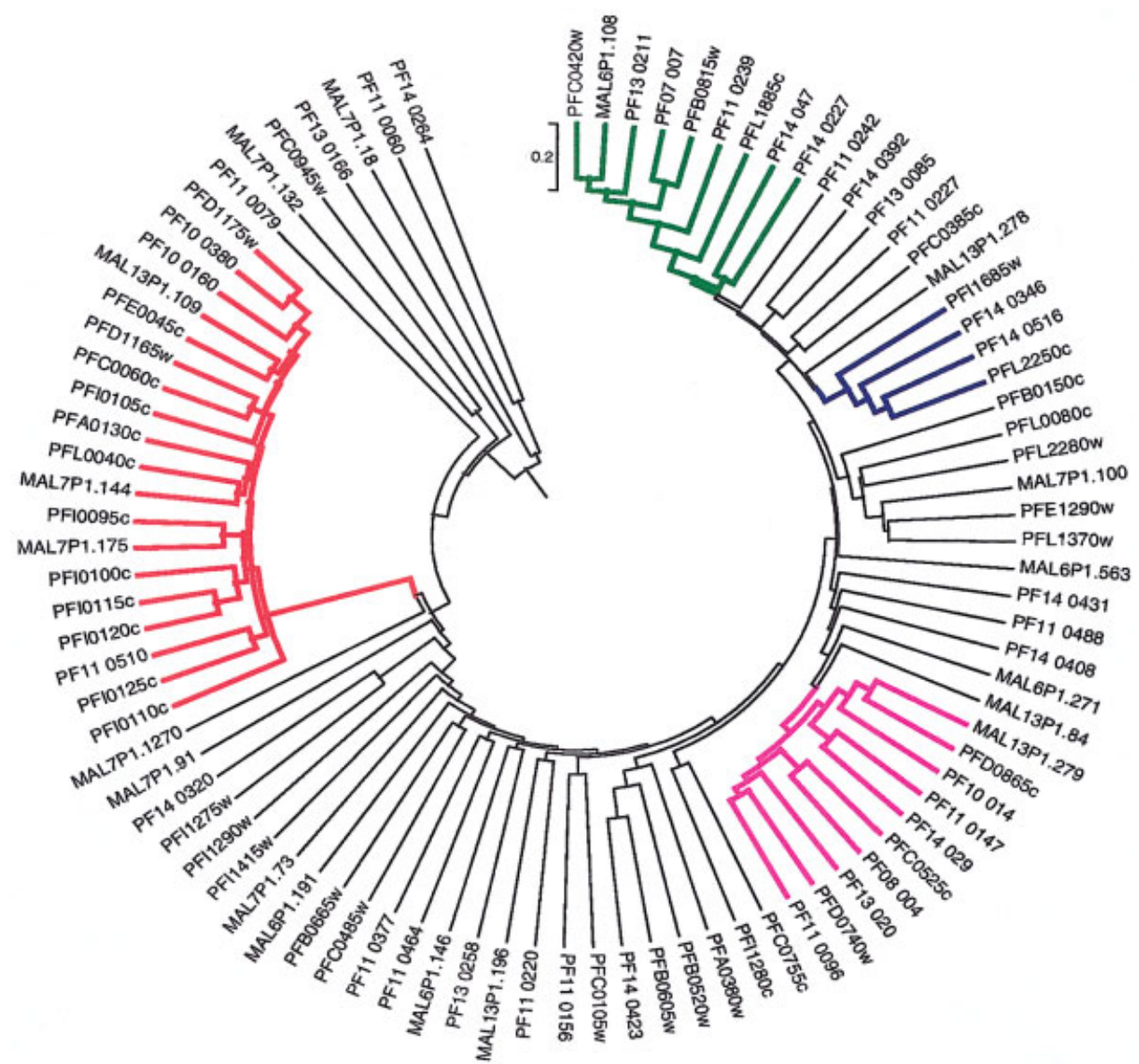

Fig. 1. Dendrogram of protein kinases in $P$. falciparum classified into known and new subfamilies. The three major subfamilies of CAMK, CMGC, AGC, and the new subfamily are coloured green, pink, blue, and red, respectively. The homologues of other groups of eukarytotic protein kinases are also shown.

domain, which is known to bind to phospholipids, therefore suggests the translocation and anchorage of the kinase to the erythrocytic membrane through this domain.

\section{MAP Kinases of $P$. falciparum}

The core components of the MAP Kinase cascade involved in stress response and cellular differentiation include $3 \mathrm{PKs}$, namely, the MAP Kinase Kinase Kinase (M3K), MAP Kinase Kinase (MEK), and the MAP Kinase (MAPK). ${ }^{43}$ The dual phosphorylation of the activation segment on threonine and tyrosine in the well-conserved TXY motif by MEK leads to the activation of MAPKs. ${ }^{44}$

Of the two MAPKs identified in the $P$. falciparum genome sharing a sequence identity of $41 \%$ in their catalytic domain, the TXY motif is completely conserved in one of them as TDY (PF11_0147), and is altered to TSH in the other (PF14_0294). It has been previously shown ${ }^{45,46}$ that the activation of the atypical MAP kinase (PF14_0294) occurs by the phosphorylation at the activation segment by Pfnek-1, a NIMA family-related kinase, and undergoes autophosphorylation in vitro. ${ }^{47}$ This atypical MAPK is 914 aa long and contains a long stretch of Gly- and Lys-rich regions. The catalytic domain of this MAPK shares a sequence identity of $41 \%$ with the other MAPK.
The MEK and MEK kinase (MEKK) homologues are, however, not identified previously in the parasite genome. We have identified a PKLS of 2987 residues long (PFB0150c) that shares greater than 30\% identity in the catalytic domain with p21-activated kinases and MEKs. This gene product has the phosphorylation site motif "SQDS" similar to the "SMAHS" signature of MEK, where the C-terminal serine is phosphorylated by the upstream MEKK. Apart from a single Raf family kinase, 2 other PKs (PF13_0258, MAL6P1.191) having closest similarity to mixed lineage kinases have been identified. These $3 \mathrm{ki}$ nases are likely to serve as upstream kinases of the putative MAPK Kinase (PFB0150c) described earlier. These observations suggest a complex interplay between the various components of MAP Kinase cascade involved in the regulation of appropriate transcription factors. The Raf kinase homologue (PF11_0079) also contains SAM (sterile alpha motif) domain and 2 MORN (membrane occupation and recognition nexus) repeat motifs that are also found in proteins occurring at membrane junctions, suggesting the perception of signals from proteins proximal to the membrane. SAM domain is also identified in one of the mixed lineage kinase (MLK) homologues (PF13_0258) of the parasite. The oligomerization through 

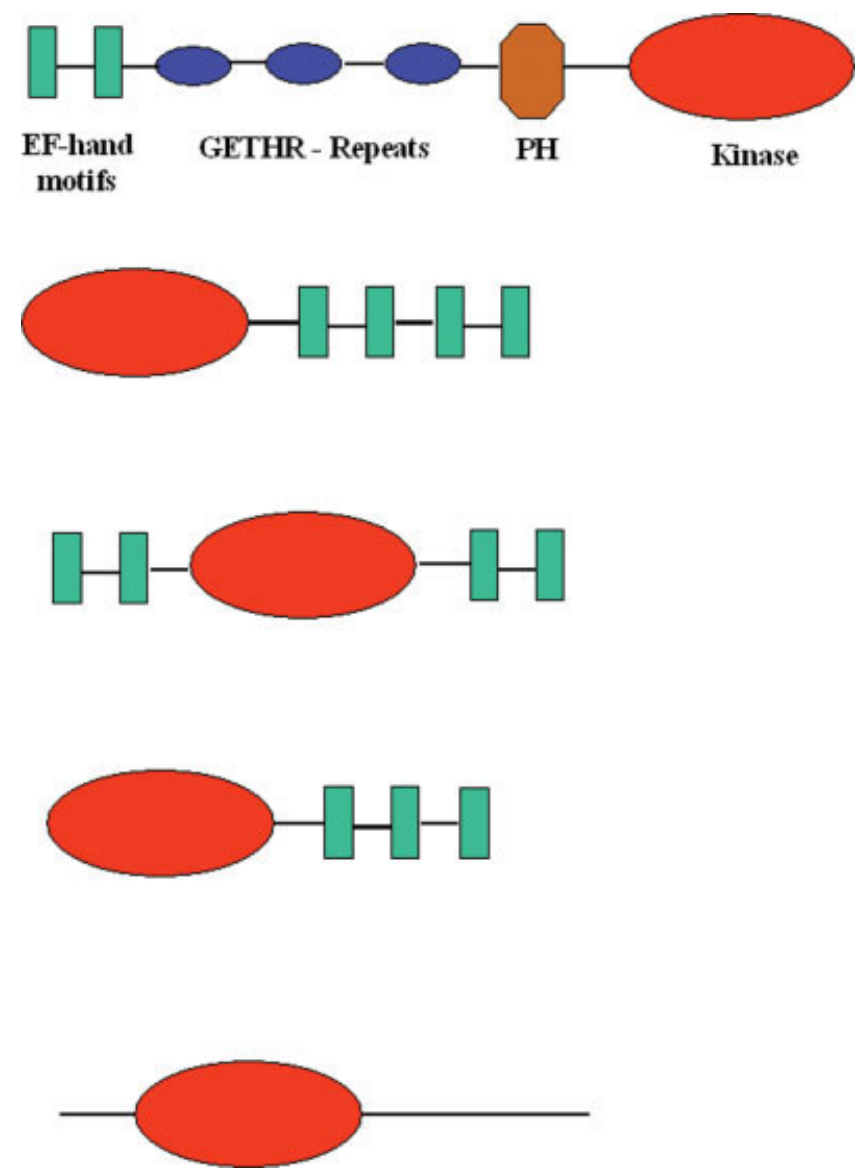

Fig. 2. The domain organization of calcium-dependent protein kinases of $P$. falciparum. Bigger ellipses indicate the protein kinase catalytic domain. The EF-hand domain (rectangular bars) binds to calcium ions. The GETHR repeats are known to occur in proteins localized to the microneme, a unique organelle found in Plasmodium species. Pleckstrin homology domain $(\mathrm{PH})$ is known to bind to phospholipids.

SAM domains is therefore likely to be important for the functioning of these kinases. The mitogen-activated protein (MAP) Kinase pathway, with these typical and atypical variants in $P$. falciparum, is therefore more divergent compared to any of the previously known MAPK pathways of other eukaryotes.

\section{Homologues of Cyclin-Dependent Kinases and Their Regulatory Proteins}

Cyclin-dependent kinases (CDKs) control the progression of the eukaryotic cell cycle in conjunction with various regulatory proteins. ${ }^{48}$ The developmental cycle of $P$. falciparum in erythrocytes differs from the classic cell cycle in leading to a nuclear polyploidy by multiple rounds of DNA replication. ${ }^{49} \mathrm{~A}$ high divergence is therefore expected in the modalities of regulation of such endoreduplication events. Previous identification of homologues of CDKs and their associated proteins ${ }^{50}$ of the parasite and the current analysis suggest that the distinct mechanisms of parasitic development, however, require the core components of the cell cycle conserved across various eukaryotes.

The parasite genome encodes at least 10 PKLSs bearing similarity in their kinase domains to the CDKs. These could be further categorized based on the extent of similarity in the cyclin-binding regions and the inhibitory phosphorylation sites into CDKs, CDK-like sequences (CLKs) and CDK-related kinases (CRKs). There are 4 putative CDKs (MAL13P1.279, PF10_0141, PF13_0206, and MAL6P1.271) that are distinguished from other CDK-like sequences in the genome by a well-conserved inhibitory phosphorylation site with minimal residue variation at the cyclin-binding regions.

In addition to the previously studied cyclin, ${ }^{51}$ Pf-cyc1 (PF14_0605), a second homologue of cyclin (PF13_0022), has been identified. The CDK-interacting residues of both the cyclins are well conserved (data not shown) and share a sequence identity of about $25 \%$. The cyclin $\mathrm{H}^{52}$ in various multicellular eukaryotes is closely related to the cyclins of the parasite (on the order of $30 \%$ sequence identity) as compared to other eukaryotic cyclins. Earlier studies have indicated Pf-cyc1 binds to the CDK1/CDK5-like homologue of the parasite and does not bind to CDK-7 homologue (Pfmrk) of P. falciparum. ${ }^{51}$

The recently available crystal structure of Plasmodium CDK (Pfpk5) ${ }^{53}$ with and without inhibitors, also reveals interesting conformational features, with the CDK adopting a structure similar to the cyclin-bound forms of CDKs in the presence of ATP analogs. Despite such conformational variations, the 2 cyclins encoded by the parasite above can potentially make analogous interactions, as reported with the 3D structures of CDK-cyclin complex ${ }^{54}$ as the critical residues involved in the interprotein contacts are all conserved (data not shown).

The Wee1 homologue of $P$. falciparum (MAL13P1.114) is a very large gene product with 3452 amino acids and lacks the catalytic aspartate in the kinase domain. The absence of catalytic aspartate in the putative homologue of Wee1 leaves open the question of the regulation of CDKs by inhibitory phosphorylation.

The cyclin-dependent kinase regulatory subunit (CKS) homologue (PFI1155w), with well-conserved residues in the binding sites of CDK and anions ${ }^{55}$ similar to other eukaryotic counterparts, has been identified. The CKS subunit is, however, larger (357 residues) than other currently known eukaryotic homologues, and is characterized by long stretches of Asn and basic amino acid-rich regions at its $\mathrm{N}$-terminal. Another group ${ }^{42}$ has also recently reported the unusual insertion in CKS. The implication of such phosphorylation to the CDK activity is, however, unclear. A putative kinase-associated phosphatase (KAP) that has been identified is known to dephosphorylate the phospothreonine on the activation segment of eukaryotic CDKs. ${ }^{56}$ All the residues involved in CDK binding and the catalytic residues are highly conserved in the KAP that shares a $24 \%$ sequence identity with human KAP. The phospho-site on the activation segment is hence suggested to switch the kinase between active and inactive states, analogous to other kinases, by phosphorylation. The other PKLSs sharing similarity with the CDK catalytic domain have a highly varied catalytic domain, with insertion and long extension at the $\mathrm{N}$ - and C-terminals of the kinase domain. Few of these CLKs have been character- 
ized and their stage-specific expression documented by other studies. ${ }^{49}$

The homologues of the CMGC subfamily of eukaryotic kinases, glycogen synthase kinase (GSK) and casein kinase-2 (CK2) have also been identified in the parasite genome. $P$. falciparum encodes 3 GSKs (MAL13P1.84, PFC0525c, and PF08_0044). They share a sequence similarity of greater than $45 \%$ with the other eukaryotic GSKs. An unusual feature of one of the GSKs is the presence of insertions of 190 aa and 330 aa between the helices $\alpha-D$ and $\alpha-\mathrm{E}$ in the $\mathrm{C}$-terminal lobe and between the catalytic loop and the activation segment, respectively. The catalytic domain therefore spans almost the entire length of the 919 aa long gene product. This gene product has been previously shown to localize at the parasite membrane during the intraerythrocytic life cycle. ${ }^{57}$

A single CK2 (PF11_0096) homologue is encoded in the parasite genome. The CK2 is known to be active as both monomer and as a tetrameric holoenzyme. The functional holoenzyme comprises 2 CK2- $\beta$ subunits and the 2 CK2- $\alpha$ (catalytic) subunits. ${ }^{58}$ Two homologues (PF11_0048 and PF13_0232) of the regulatory subunit CK2- $\beta$ have also been identified in $P$. falciparum that share greater than $35 \%$ identity with the human CK2 $-\beta$ subunit. The 2 homologues share a sequence identity of $36 \%$ and long insertions of about $30 \mathrm{Glu}$ and $20 \mathrm{Asp}$ lie in the N-terminal region of one of the homologues (PF13_0232).

\section{AGC Subfamily of Protein Kinases in $P$. falciparum}

The binding of second messengers, like cyclic adenosine monphosphate (cAMP), cyclic guanosine monophosphate (cGMP), and diacyl glycerol (DAG) regulates the PK members of the protein kinase A, protein kinase $G$, and protein kinase C (AGC) family. ${ }^{1}$ The parasite genome encodes 5 PKLSs that are closely related to the catalytic domain of AGC kinases. The PKG homologue (PF14_0346), with $4 \mathrm{cNMP}$-binding domains located at the $\mathrm{N}$-terminal of the kinase domain, has been identified. This protein kinase $\mathrm{G}$ (PKG) has also been shown to express in the intraerythrocytic asexual stage of $P$. falciparum and is known to vary with respect to nucleotide-binding affinity. ${ }^{59}$ The other eukaryotic homologues have only $2 \mathrm{cNMP}$ binding domains tethered to the catalytic domain. Two putative homologues of PKA (PF11_0227 and PF11_685w) and a single regulatory subunit of PKA (PFL1110c) have been identified in $P$. falciparum. One of the PKAs has been previously shown to be required for the development of the parasite. $^{60}$

Protein kinase C (PKC) homologues have not been identified in the parasite. A single PKLS that is closest in terms of sequence similarity to PKC (PFL2250c) lacks the calcium- and phospholipid-binding domains that are characteristic of the PKC family of PKs. The CDPK homologue (PF11_0242), with EF-hands and PH domain, may perform functions analogous to mammalian PKCs that are activated by calcium and phospholipids.

\section{Other P. falciparum Protein Kinases}

The NIMA family of enzymes plays an important role in the eukaryotic cell cycle division processes involving chro- matin condensation and formation of mitotic spindles. ${ }^{61}$ Four homologues of the NIMA family kinases have been identified. One member of the family, Pfnek-1 (PFL1370w), is shown to be analogous to eukaryotic MEK as it phosphorylates the atypical MAP Kinase described in the previous sections. The Pfnek-1 also has a phosphorylation site similar to MEK, characterized by the motif "SMAHS" replacing the NIMA family kinase motif of "FXXT." In the other 3 NIMA homologues, the "FXXT" motif is partially conserved as "YXXT" (MAL7P1.100) and "QXXT" (PFE1290w), where the threonine at the end of the motif is phosphorylated by various kinases. The motif is quite distinct in another homologue, where the position of phospho site is not conserved and is altered to "SSNN." Hence, the NIMA family kinases of the parasite appear to have diverged highly in terms of sequence similarity, regulation, and interacting proteins from their other eukaryotic counterparts known so far.

The plant receptor kinase-like (RLK) family ${ }^{62}$ is another plant characteristic family of PKs in addition to the CDPKs that are encoded in $P$. falciparum. A single homologue (PF11_0220) of this family with GETHR pentapeptide repeats commonly occurring in proteins localized in microneme, a subcellular organelle of Plasmodium has also been identified. The RLK does not contain any transmembrane-spanning segments.

Two homologues of PKs of eukaryotes (MAL6P1.146 and PF14_0423) involved in the regulation of translation by phosphorylation of eukaryotic translation-initiation factor-2 (eIF-2 $\alpha$ ) have also been identified. The presence of heme-dependent eIF2-kinase activity is previously demonstrated in the parasite, ${ }^{63}$ suggesting the existence of a heme-regulated inhibitory (HRI) class of eIF2 kinase. One of these gene products identified in the current analysis, PfPK4 (MAL6P1.146), has been well characterized previously. ${ }^{64}$ The previously sequenced gene product, Pfpk4, of the parasite is 1123 aa long, while the corresponding sequence in the database of ORFs of the $P$. falciparum is 3072 amino acids long, sharing 94\% identity. A 1907-residuelong N-terminal region of the translated ORF is missing in the PfPK4. A putative transmembrane region is also predicted at the N-terminal of the gene product. Hence, it is currently unclear if the PfPK4 and the gene product (MAL6P1.146) are alternate spliced forms of the same gene.

Another member of the eIF2-kinase subgroup (PF14_0423) is 1553 amino acids long, with the kinase catalytic domain located in the middle of the gene product and long extensions at $\mathrm{C}$ - and N-terminal ends. The catalytic domain of these 2 kinases has insertions (about 100 aa in PF14_0423 and about 500 aa in MAL6P1.146) larger than previously known for this family of kinases.

A PK (PF14_0320) identified in the parasite contains 6 putative membrane-spanning segments. The membranespanning regions are located at the N-terminal of the kinase domain. An extracellular region at the $\mathrm{N}$-terminal is about 130 residues long and lacks similarity to protein domains of known function. 


\section{A New Subfamily of Protein Kinases}

The PKs of $P$. falciparum that could not be associated to any known subfamilies were clustered together based on their sequence similarity, as described in the Materials and Methods section. A single large cluster of 19 PKLSs has been identified. Most of the kinases in this cluster are encoded as gene products of similar lengths (550-700 aa), with the kinase domain located at the C-terminal. The kinase domains of the gene products in the new group share an identity of $40 \%$. In addition, a region of 120-150 residues located $\mathrm{N}$-terminal to the kinase domain is also found to be conserved in all the members of this group. However, similarity to any known protein domain families could not be detected for this region. This new subfamily of PKs identified in P. falciparum is therefore suggested to be the most highly divergent class of PKs known so far. All the kinases of this group (Fig. 3) are non-RD kinases, suggesting a phosphorylation (at activation segment) independent regulation.

The kinase catalytic domain of members of the new subfamily of PKs has been further used as a query, to search the NRDB database in order to identify their closest homologues from other species. The highest sequence identity of the closest homologue (gi|40557180|gb|AAR87861.1 of another apicomplexan species, Cryptosporidium parvum) with any of the new subfamily members of PKLSs is $35 \%$. A phylogenetic analysis, based on the sequence similarity between the kinase domains of the new members and their closest homologues from other species, reveals distinct grouping of the new subfamily of kinases (Fig. 3). These observations suggest that the $19 \mathrm{PKs}$ of $P$. falciparum constitute a new subfamily of PKs that are uniquely conserved in Plasmodium. Their functional roles in the life cycle of the parasite remains to be elucidated experimentally. The expression data of the ORFs of only 4 of the new subfamily members (PFI0095c, PFA0130c, MAL13P1.109, and PFE0045c) are currently unavailable.

\section{Kinase Inserts of $P$. falciparum}

The catalytic domain of a significant number of PKLSs has insertions that are not characteristic of eukaryotic PKs known so far. These insertions are also observed in the PK homologues of CMGC group and Plant Receptor Kinase subfamily. Most of these insertions are in the loops linking various secondary structures [Fig. 4(a)], and their lengths vary in the range of 10-500 residues [Fig. 4(b)]. The kinase catalytic core is hence suggested to accommodate such large insertions in between the regular secondary structures.

\section{Protein Kinases Translocated to Apicoplast}

The apicoplast of Plasmodium is a small organelle that houses many critical enzymes and is known to be indispensable for the invasion of new host cells by the parasite. Various proteins translocated to the apicoplast are guided by a bipartite signal comprising a signal peptide recruiting them to secretory pathways, followed by a transit peptide. ${ }^{30,31}$ Putative apicoplast targeting sequences have been identified, as described in the
Materials and Methods section, at the N-terminal end of $11 \mathrm{PKs}$. While a few positively charged residues are conserved in most of these transit peptides, the nature of other residues is highly variable. The list of these PKs is provided (supplementary information 2). Another kinase, referred to as Falciparum exported serine/threonine kinase (FEST, MAL7P1.91), is known to be exported $^{65}$ from the parasite into the host cell.

\section{DISCUSSION}

With the exception of the tyrosine kinase family, comparisons of the PK repertoires in eukaryotes ${ }^{14,35-39}$ suggest the occurrence of all the known subfamilies, while the extent of expansion of a given subfamily varies in different organisms. Saccharomyces cerevisiae lacks PKs belonging to the protein tyrosine kinase subfamilies.

However, the $P$. falciparum genome lacks homologues of PKC-like, P-21-activated kinase, typical MEK, MEKKs, and receptor tyrosine kinases. In addition, the homologues of the PK subfamilies known so far and conserved across eukaryotic organisms are longer in the parasite, due to insertions in the catalytic domain and/or the presence of long stretches of polar and charged residues of yet unknown functions.

In comparison to $S$. cerevisiae, the parasite genome encodes 2 additional subfamilies that are characteristic of plant kinases that include CDPKs and plant receptor kinase. Thus, a distinct repertoire of the parasite PKs suggests a highly divergent evolution of this family of PKs known so far among various eukaryotic organisms.

The current survey for PKs in the parasite genome has revealed the distinct nature of the parasitic PKs. The PKs of the parasite are encoded by approximately $2 \%$ of the total genome, as in other eukaryotes. Receptor tyrosine kinases are absent in the parasite, consistent with the absence of tyrosine kinases in $S$. cerevisiae, another unicellular eukaryote. With the exception of CDKs, the sequence similarity between Plasmodium PKs and other eukaryotic kinases known so far is very limited.

The CDPK family is highly represented in the parasite. Identification of a CDPK with $\mathrm{PH}$ and EF-hands is also suggestive of a PKC-like enzyme in terms of probable regulation by calcium and phospholipids. The MAP kinase pathway also appears to deviate from the canonical cascades, with the participation of NIMA-like kinases and highly divergent MEKs in their signaling pathways. Homologues of the CDKs and their regulatory proteins, including a new cyclin, CKS, and KAP revealed from the analysis indicate employment of conserved signaling components in the cell-cycle machinery across diverse organisms. Occurrences of RLK sequences without a membranespanning segment and CDPKs suggest a close evolutionary link between the plant and parasite kinases of the two subfamilies. The PKs of the new subfamily identified in the analysis share high sequence similarity in the catalytic and nonkinase regions, suggesting functional features common to the members of this subfamily.

In contrast to $S$. cerevisiae, the parasite encodes a PK (PF14_0320) with transmembrane-spanning segments. 


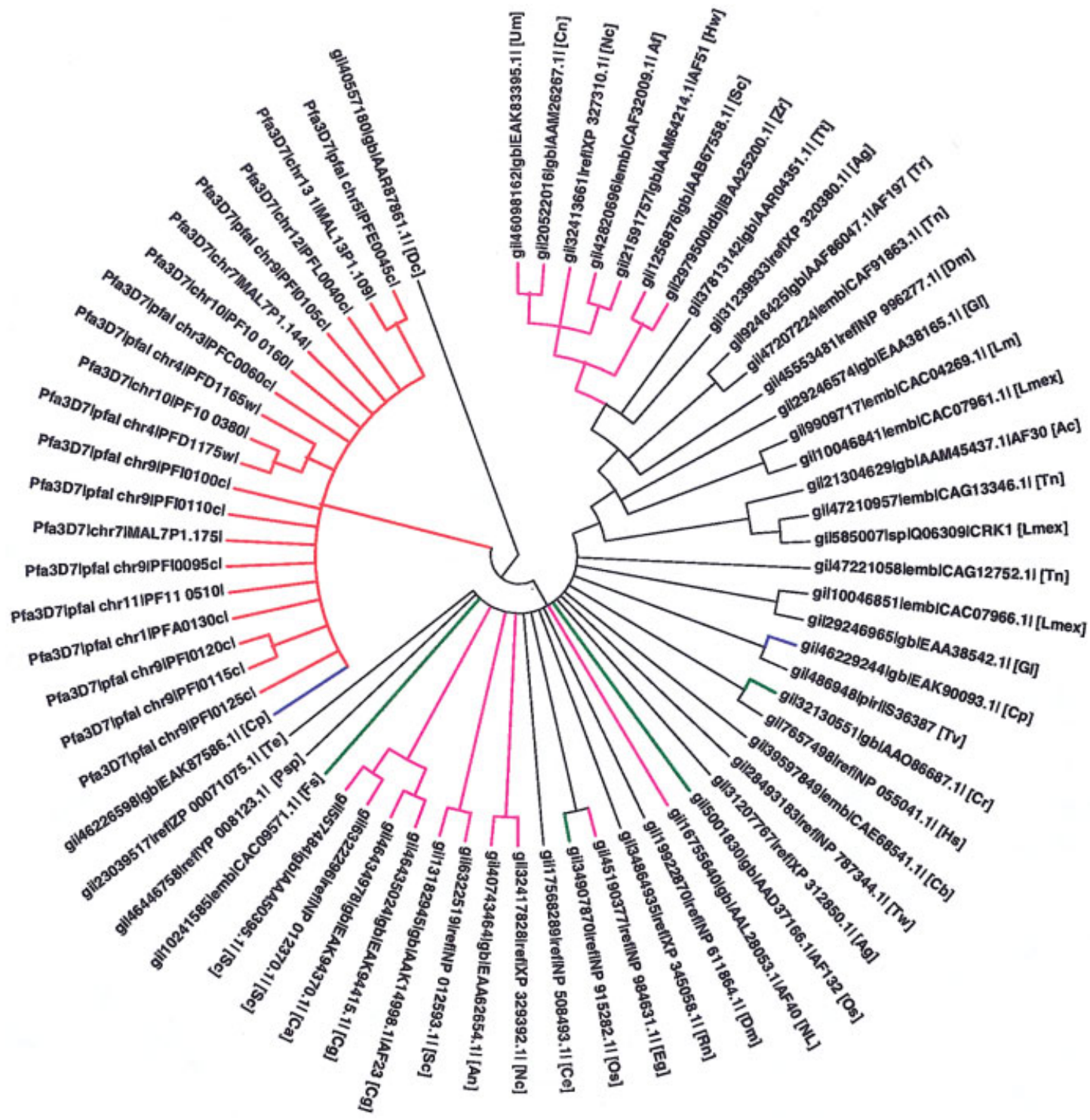

Fig. 3. The dendrogram based on the sequence similarity of the protein kinases of $P$. falciparum constituting the new subfamily and their closest homologues is shown. New family members are indicated in red. Homologues of other apicomplexan species, plants, and fungi are colored blue, green, and pink, respectively. Species abbreviations followed in the dendrogram: An, Aspergillus nidulans; $\mathrm{Cp}$, Cryptosporidium parvum; Te, Trichodesmium erythraeum; Lmex, Leishmania mexicana; Lm, Leishmania major, Psp, Parachlamydia species; Sc, Saccharomyces cerevisiae; Dm, Drosophila melanogaster, Af, Aspergillus fumigatus; Cn, Cryptococcus neoformans; Cr, Chalamydomonas reinhardti;; Rn, Rattus norvegicus; Tt, Tetrahymena thermophila; Tr, Takifugu rubripes; Tn, Tetraodon nigroviridis; Tw, Tropheryma whipplei; Gl, Giardia lamblia; $\mathrm{Ag}$, Anopheles gambiae; Ca, Candida albicans; Hs, Homo sapiens; Zr, Zygosaccharomyces rouxii; Um, Ustilago maydis; Eg, Eremothecium gossypii; Nc, Neurospora crassa; Hw, Hortaea werneckii; Dc, Dinocampus coccinellae; Nl, Nosema locustae; Cb, Caenorhabditis briggsae; Cg, Candida glabrata; Ac, Axinella corrugata; Fs, Fagus sylvatica; Os, Oryza sativa; Ce, Caenorhabditis elegans; Tv, Trichomonas vaginalis.

This new kinase is the first of the eukaryotic kinases known to have 6 putative membrane-spanning regions, suggesting a unique receptor-like function.

High divergence of PKs is also indicated by the occurrences of PKs of unusual lengths, with insertions in the catalytic domain. The location of insertions in kinases between well-conserved secondary structures suggests the high flexibility of the kinase catalytic core to accommodate large inserts, with minimum disruption to the catalytic domain. These insertion regions might also serve as substrate-binding or regulatory sites.

The PKs of $P$. falciparum therefore represent a highly divergent class of PKs that are distinct in various features from other eukaryotic kinases known so far. We hope that the variant modular organization and occurrence of atypical features in the PKs of parasites, as revealed in the analysis, therefore lead to experimental studies exploring their biological roles and regulatory processes. 


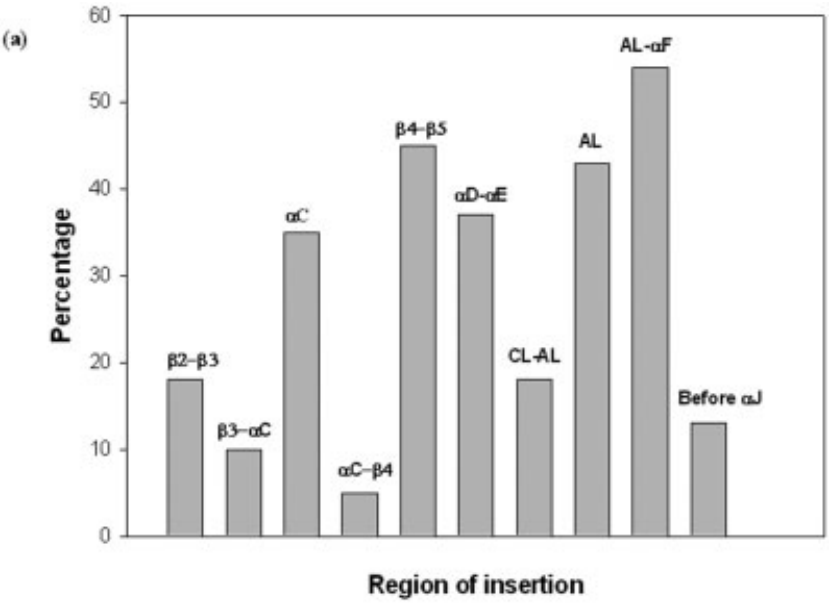

(b)

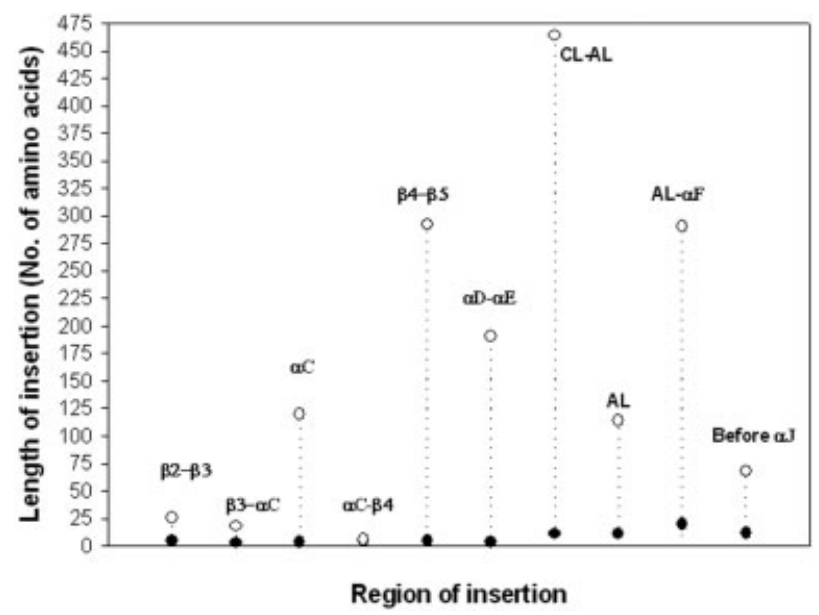

Fig. 4. The locations and lengths of inserts of protein kinases of $P$. falciparum having insertions (37 PKLSs) in the catalytic domain. (a) The normalized frequency of occurrences of inserts at various regions in the catalytic core of $P$. falciparum protein kinases possessing large inserts. (b) The range of lengths of the inserts occurring in the catalytic core: $\alpha$, $\alpha$-helix; $\beta, \beta$-strand. The numerals and the letter of the alphabet following each secondary structure element follow the numbering as defined for the 3D structure of protein kinase A (2). Abbreviations: CL, catalytic loop; AS, activation segment. The minimum and maximum size of insertions are indicated by filled and open circles, respectively.

\section{ACKNOWLEDGMENTS}

Our thanks to K. R. Abhinandan for his help in the preparation of the online information about $P$. falciparum kinases, and to $\mathrm{B}$. Anand for providing data regarding the benchmarking parameters of various sequence and profile search methods.

\section{REFERENCES}

1. Hanks SK, Quinn AM, Hunter T. The protein kinase family: conserved features and deduced phylogeny of the catalytic domains. Science 1998;241:42-52.

2. Zheng J, Knighton DR, ten Eyck LF, Karlsson R, Xuong N, Taylor SS, Sowadski JM. Crystal structure of the catalytic sub-unit of cAMP dependent protein kinase complexed with MgATP and peptide inhibitor. Biochemistry 1993;32:2154-2161.
3. Johnson LN, Noble ME, Owen DJ. Active and inactive protein kinases: structural basis for regulation. Cell 1996;85:149-158.

4. Dluzewski AR, Garcia CR. Inhibition of invasion and intraerythrocytic development of Plasmodium falciparum by kinase inhibitors. Experientia 1996;52:621-623.

5. Magowan C, Liang J, Yeung J, Takakuwa Y, Coppel RL, Mohandas N. Plasmodium falciparum: influence of malarial and host erythrocyte skeletal protein interactions on phosphorylation in infected erythrocytes. Exp Parasitol 1998;89:40-49.

6. Zhao Y, Franklin RM, Kappes B. Plasmodium falciparum calciumdependent protein kinase phosphorylates proteins of the host erythrocytic membrane. Mol Biochem Parasitol 1994;66:329-343.

7. Jones GL, Edmundson HM. Protein phosphorylation during the asexual life cycle of the human malarial parasite Plasmodium falciparum. Biochim Biophys Acta 1990;1053:118-124.

8. Gardner MJ, Hall N, Fung E, White O, Berriman M, Hyman RW, Carlton JM, Pain A, Nelson KE, Bowman S, Paulsen IT, James K, Eisen JA, Rutherford K, Salzberg SL, Craig A, Kyes S, Chan MS, Nene V, Shallom SJ, Suh B, Peterson J, Angiuoli S, Pertea M, Allen J, Selengut J, Haft D, Mather MW, Vaidya AB, Martin DM, Fairlamb AH, Fraunholz MJ, Roos DS, Ralph SA, McGadden GI, Cummings LM, Subramanian GM, Mungall C, Venter JC, Carucci DJ, Hoffman SL, Newbold C, Davis RW, Fraser CM, Barrell B. Genome sequence of the human malaria parasite Plasmodium falciparum. Nature 2002;419:498-511.

9. Bahl A, Brunk B, Crabtree J, Fraunholz MJ, Gajria B, Grant GR, Ginsburg H, Gupta D, Kissinger JC, Labo P, Li L, Mailman MD, Milgram AJ, Pearson DS, Roos DS, Schug J, Stoeckert CJ Jr, Whetzel P. PlasmoDB: the Plasmodium genome resource: a database integrating experimental and computational data. Nucleic Acids Res 2003;31:212-215.

10. Pertea M, Salzberg SL, Gardner MJ. Finding genes in Plasmodium falciparum. Nature 2000;404:34.

11. Lawson D, Bowman S, Barrell B. Finding genes in Plasmodium falciparum. Nature 2000;404:34-35.

12. Salzberg SL, Pertea M, Delcher AL, Gardner MJ, Tettelin H. Interpolated Markov models for eukaryotic gene finding. Genomics 1999;59:24-31.

13. Cawley SE, Wirth AI, Speed TP. Phat-a gene finding program for Plasmodium falciparum. Mol Biochem Parasitol 2001;118:167174.

14. Krupa A, Srinivasan N. The repertoire of protein kinases encoded in the draft version of the human genome: atypical variations and uncommon domain combinations [Research 0066]. Genome Biol 2002;3:1-14.

15. Krupa A, Abhinandan KR, Srinivasan N. KinG: a database of protein kinases in genomes. Nucleic Acids Res 2004;32:D153D155.

16. Altschul SF, Madden TL, Schaffer AA, Zhang J, Zhang Z, Miller W, Lipman DJ. Gapped BLAST and PSI-BLAST: a new generation of protein database search programs. Nucleic Acids Res 1997;25: $3389-3402$.

17. Marchler-Bauer A, Anderson JB, DeWeese-Scott C, Fedorova ND, Geer LY, He S, Hurwitz DI, Jackson JD, Jacobs AR, Lanczycki CJ, Liebert CA, Liu C, Madej T, Marchler GH, Mazumder R, Nikolskaya AN, Panchenko AR, Rao BS, Shoemaker BA, Simonyan V, Song JS, Thiessen PA, Vasudevan S, Wang Y, Yamashita RA, Yin JJ, Bryant SH. CDD: a curated Entrez database of conserved domain alignments. Nucleic Acids Res 2003;31:383-387.

18. Eddy SR. Profile hidden Markov models. Bioinformatics 1998;14: 755-763.

19. Lindahl E, Elofsson A. Identification of related proteins on family, superfamily and fold level. J Mol Biol 2000;295:613-625.

20. Andreeva A, Howorth D, Brenner SE, Hubbard TJ, Chothia C, Murzin AG. SCOP database in 2004: refinements integrate structure and sequence family data. Nucleic Acids Res 2004;32:D226 D229.

21. Gowri VS, Pandit SB, Karthik PS, Srinivasan N, Balaji S. Integration of related sequences with protein three-dimensional structural families in an updated version of PALI database. Nucleic Acids Res 2003;31:486-488.

22. Muller A, MacCallum RM, Sternberg MJ. Benchmarking PSI-BLAST in genome annotation. J Mol Biol 1999;293:1257-1271.

23. Le Roch KG, Zhou Y, Blair PL, Grainger M, Moch JK, Haynes JD, De La Vega P, Holder AA, Batalov S, Carucci DJ, Winzeler EA. Discovery of gene function by expression profiling of the malaria parasite life cycle. Science 2003;301:1503-1508. 
24. Florens L, Washburn MP, Raine JD, Anthony RM, Grainger M, Haynes JD, Moch JK, Muster N, Sacci JB, Tabb DL, Witney AA, Wolters D, Wu Y, Gardner MJ, Holder AA, Sinden RE, Yates JR, Carucci DJ. A proteomic view of the Plasmodium falciparum life cycle. Nature 2002;419:520-526.

25. Lasonder E, Ishihama Y, Andersen JS, Vermunt AMW, Pain A, Sauerwein RW, Eling WMC, Hall N, Waters AP, Stunnenberg HG, Mann M. Analysis of the Plasmodium falciparum proteome by high-accuracy mass spectrometry. Nature 2002;419:537-542.

26. Chenna R, Sugawara H, Koike T, Lopez R, Gibson TJ, Higgins DG, Thompson JD. Multiple sequence alignment with the Clustal series of programs. Nucleic Acids Res 2003;31:3497-3500.

27. Kumar S, Tamura K, Nei M. MEGA: Molecular Evolutionary Genetics Analysis software for microcomputers. Comput Appl Biosci 1994;10:189-191.

28. Bateman A, Birney E, Cerruti L, Durbin R, Etwiller L, Eddy SR, Griffiths-Jones S, Howe KL, Marshall M, Sonnhammer EL. The Pfam protein families database. Nucleic Acids Res 2002;30:276280

29. Krogh A, Larsson B, von Heijne G, Sonnhammer EL. Predicting transmembrane protein topology with a hidden Markov model: application to complete genomes. J Mol Biol 2001;305:567-580.

30. Foth BJ, Ralph SA, Tonkin CJ, Struck NS, Fraunholz M, Roos DS, Cowman AF, McFadden GI. Dissecting apicoplast targeting in the malaria parasite Plasmodium falciparum. Science 2003;299:705708.

31. Zuegge J, Ralph S, Schmuker M, McFadden GI, Schneider G. Deciphering apicoplast targeting signals-feature extraction from nuclear-encoded precursors of Plasmodium falciparum apicoplast proteins. Gene 2001;280:19-26.

32. Potter LR, Hunter T. Phosphorylation of the kinase homology domain is essential for activation of the A-type natriuretic peptide receptor. Mol Cell Biol 1998;18:2164-2172.

33. Kroiher M, Miller MA, Steele RE. Deceiving appearances: signaling by "dead" and "fractured" receptor protein-tyrosine kinases. Bioessays 2001;23:69-76.

34. Papa FR, Zhang C, Shokat K, Walter P. Bypassing a kinase activity with an ATP-competitive drug. Science 2003;302:15331537.

35. Hunter T, Plowman GD. The protein kinases of budding yeast: six score and more. Trends Biochem Sci 1997;22:18-22.

36. Plowman GD, Sudarsanam S, Bingham J, Whyte D, Hunter T. The protein kinases of Caenorhabditis elegans: a model for signal transduction in multicellular organisms. Proc Natl Acad Sci USA 1999;96:13603-13610.

37. Morrison DK, Murakami MS, Cleghon V. Protein kinases and phosphatases in the Drosophila genome. J Cell Biol 2000;150:F57F62.

38. Kostich M, English J, Madison V, Gheyas F, Wang L, Qiu P, Greene J, Laz TM. Human members of the eukaryotic protein kinase family [Research 0043]. Genome Biol 2002;3:1-12.

39. Manning G, Whyte DB, Martinez R, Hunter T, Sudarsanam S. The protein kinase complement of the human genome. Science 2002;298:1912-1934.

40. Cheng SH, Willmann MR, Chen HC, Sheen J. Calcium signaling through protein kinases: the Arabidopsis calcium-dependent protein kinase gene family. Plant Physiol 2002;129:469-485.

41. Read LK, Mikkelsen RB. Cyclic AMP- and Ca2(+)-dependent protein kinases in Plasmodium falciparum. Exp Parasitol 1990;71: $39-48$.

42. Aravind L, Iyer LM, Wellems TE, Miller LH. Plasmodium biology: genomic gleanings. Cell 2003;115:771-785.

43. Lewis TS, Shapiro PS, Ahn NG. Signal transduction through MAP kinase cascades. Adv Cancer Res 1998;74:49-139.

44. Boulton TG, Nye SH, Robbins DJ, Ip NY, Radziejewska E, Morgenbesser SD, DePinho RA, Panayotatos N, Cobb MH, Yancopoulos GD. ERKs: a family of protein-serine/threonine kinases that are activated and tyrosine phosphorylated in response to insulin and NGF. Cell 1991:65:663-675.

45. Lin DT, Goldman ND, Syin C. Stage-specific expression of a
Plasmodium falciparum protein related to the eukaryotic mitogenactivated protein kinases. Mol Biochem Parasitol 1996;78:67-77.

46. Dorin D, Le Roch K, Sallicandro P, Alano P, Parzy D, Poullet P, Meijer L, Doerig C. Pfnek-1, a NIMA-related kinase from the human malaria parasite Plasmodium falciparum: biochemical properties and possible involvement in MAPK regulation. Eur J Biochem 2001;268:2600-2608.

47. Dorin D, Alano P, Boccaccio I, Ciceron L, Doerig C, Sulpice R, Parzy D, Doerig C. An atypical mitogen-activated protein kinase (MAPK) homologue expressed in gametocytes of the human malaria parasite Plasmodium falciparum: identification of a MAPK signature. J Biol Chem 1999;274:29912-29920.

48. Morgan DO. Cyclin-dependent kinases: engines, clocks, and microprocessors. Annu Rev Cell Dev Biol 1997;13:261-291.

49. Doerig C, Chakrabarti D, Kappes B, Matthews K. The cell cycle in protozoan parasites. Prog Cell Cycle Res 2000;4:163-183.

50. Doerig C, Endicott J, Chakrabarti D. Cyclin-dependent kinase homologues of Plasmodium falciparum. Int J Parasitol 2002;32: $1575-1585$.

51. Le Roch K, Sestier C, Dorin D, Waters N, Kappes B, Chakrabarti D, Meijer L, Doerig C. Activation of a Plasmodium falciparum cdc2-related kinase by heterologous p25 and cyclin $\mathrm{H}$ : functional characterization of a P. falciparum cyclin homologue. J Biol Chem 2000;275:8952-8958.

52. Kim KK, Chamberlin HM, Morgan, DO, Kim SH. Threedimensional structure of human cyclin $\mathrm{H}$, a positive regulator of the CDK-activating kinase. Nat Struct Biol 1996;3:849-855.

53. Holton S, Merckx A, Burgess D, Doerig C, Noble M, Endicott J. Structures of $P$. falciparum PfPK5 test the CDK regulation paradigm and suggest mechanisms of small molecule inhibition. Structure (Camb) 2003;11:1329-1337.

54. Russo AA, Jeffrey PD, Pavletich NP. Structural basis of cyclindependent kinase activation by phosphorylation. Nat Struct Biol 1996;3:696-700.

55. Parge HE, Arvai AS, Murtari DJ, Reed SI, Tainer JA. Human CksHs2 atomic structure: a role for its hexameric assembly in cell cycle control. Science 1993;262:387-395.

56. Song H, Hanlon N, Brown NR, Noble ME, Johnson LN, Barford D. Phosphoprotein-protein interactions revealed by the crystal structure of kinase-associated phosphatase in complex with phosphoCDK2. Mol Cell 2001;7:615-626.

57. Kappes B, Yang J, Suetterlin BW, Rathgeb-Szabo K, Lindt MJ, Franklin RM. Plasmodium falciparum protein kinase with two unusually large kinase inserts. Mol Biochem Parasitol 1995;72: 163-178.

58. Niefind K, Guerra B, Ermakowa I, Issinger OG. Crystal structure of human protein kinase CK2: insights into basic properties of the CK2 holoenzyme. EMBO J 2001;20:5320-5331.

59. Deng W, Parbhu-Patel A, Meyer DJ, Baker DA. The role of two novel regulatory sites in the activation of the cGMP-dependent protein kinase from Plasmodium falciparum. Biochem J 2003;374: $559-565$.

60. Li J, Cox LS. Isolation and characterisation of a cAMP-dependent protein kinase catalytic subunit gene from Plasmodium falciparum. Mol Biochem Parasitol 2000;109:157-163.

61. Fry AM, Nigg EA. Characterization of mammalian NIMA-related kinases. Methods Enzymol 1997;283:270-282.

62. Morris ER, Walker JC. Receptor-like protein kinases: the keys to response. Curr Opin Plant Biol 2003;6:339-342.

63. Surolia N, Padmanaban G. Chloroquine inhibits heme-dependent protein synthesis in Plasmodium falciparum. Proc Natl Acad Sci USA 1991;88:4786-4790.

64. Mohrle JJ, Zhao Y, Wernli B, Franklin RM, Kappes B. Molecular cloning, characterization and localization of PfPK4, an eIF-2alpha kinase-related enzyme from the malarial parasite Plasmodium falciparum. Biochem J 1997;328:677-687.

65. Kun JF, Hibbs AR, Saul A, McColl DJ, Coppel RL, Anders RF. A putative Plasmodium falciparum exported serine/threonine protein kinase. Mol Biochem Parasitol 1997;85:41-51. 